\title{
ON TWISTED LIFTING
}

\author{
BY
}

YUVAL Z. FLICKER

\begin{abstract}
If $\sigma$ is a generator of the galois group of a finite cyclic extension $E / F$ of local or global fields, and $\varepsilon$ is a character of $C_{E}\left(=E^{\times}\right.$or $\left.E^{\times} \backslash \mathbf{A}^{\times}\right)$whose restriction to $C_{F}$ has order $n$, then the irreducible admissible or automorphic representations $\pi$ of $\mathrm{GL}(n)$ over $E$ with ${ }^{\sigma} \pi \cong \pi \otimes \varepsilon$ are determined.
\end{abstract}

Let $F$ be a local or global field, and $E$ a cyclic extension of $F$ of degree $e \geq 1 ; C_{F}$ denotes the multiplicative group $F^{\times}$if $F$ is local and the idèle class group $F^{\times} \backslash \mathbf{A}^{\times}$if $F$ is global. Put $G=\mathrm{GL}_{n}$ and fix a character $\varepsilon$ of $C_{E}$ whose restriction to $C_{F}$ is of order $n$. It determines a character $\varepsilon$ of $G(E)$ or $G(E) \backslash G\left(\mathbf{A}_{E}\right)$ by $\varepsilon(g)=\varepsilon(\operatorname{det} g)$. The restriction of $\varepsilon$ to $C_{F}$ determines by class field theory a cyclic extension $F_{\varepsilon}$ of $F$ of degree $n$. Fix a generator $\sigma$ of the galois group $\operatorname{Gal}(E / F)$. Denote by $R(G)$ the set of equivalence classes of irreducible admissible (or automorphic) representations of $G(E)$ (or $G\left(\mathbf{A}_{E}\right)$ ). Then $\sigma$ acts on $R(G)$ by ${ }^{\sigma} \pi(g)=\pi(\sigma(g))$ ( $g$ in $G(E)$ or $\left.G\left(\mathbf{A}_{E}\right)\right)$, and we denote by ${ }^{\sigma} R^{\varepsilon}(G)$ the set of $\pi$ in $R(G)$ with ${ }^{\sigma} \pi \cong \pi \otimes \varepsilon$.

THEOREM. There exists a natural bijection between ${ }^{\sigma} R^{\varepsilon}(G)$ and the set of $\operatorname{Gal}\left(F_{\varepsilon} / F\right)$-orbits of characters on $N_{L / F_{\varepsilon}} C_{L}$, where $L=F_{\varepsilon} E$.

In the case $E=F$ this is due to Kazhdan [K] in the local case and if $\pi$ is cuspidal with two supercuspidal components. Kazhdan's proof is remarkably elegant. Complete local and global results are derived from the study of the unramified case only. Our proof follows Kazhdan's, and uses Kottwitz's base change lemma [Ko] and Arthur's explicit trace formula $\left[\mathbf{A}^{\prime}\right]$.

We also show (Lemma 15.1) that the above $\pi$ are induced $I_{P}^{G}(\tau)$ from a representation $\tau=\left(\tau^{\prime},{ }^{\sigma} \tau^{\prime} \otimes \varepsilon^{\prime}, \ldots,{ }^{\sigma^{m-1}} \tau^{\prime} \otimes \varepsilon^{\prime}{ }^{\sigma} \varepsilon^{\prime} \ldots{ }^{\sigma^{m-2}} \varepsilon^{\prime}\right)$, where $\varepsilon^{\prime}=\varepsilon^{-1}$ and $\tau^{\prime}$ is a (super) cuspidal representation of $\mathrm{GL}\left(\mathrm{m}^{\prime}, E\right), n=m m^{\prime}$. $P$ is a parabolic subgroup with Levi component $\mathrm{GL}\left(m^{\prime}\right)^{m}$. Then if $L^{\prime}=E \cap F_{\varepsilon}$ is an extension of $F$ of degree $l$, we must have that $l$ divides $m$ (Corollary 16.2) (and $m / l$ is prime to $e$ (Proposition 17.1)). For example, in the special case where $E=F_{\varepsilon}$, each $\pi$ of the above type is induced from a character of a Borel subgroup. Hence it is not in the discrete series. We proved this result in $[\mathbf{F}]$ when $E=F_{\varepsilon}$ is a quadratic extension of $F$, using the trace formula twisted as here. In fact this motivated us to look for a twisted analogue of Kazhdan's lifting. Further description of our $\pi$ is given in $\S \S 16$ and 17.

Received by the editors June 30, 1984.

1980 Mathematics Subject Classification. Primary 10D40. 
We restrict our attention to the case where the characteristic of $F$ is 0 . The case of char $F \neq 0$ follows from a forthcoming general method of Kazhdan.

I am grateful to D. Kazhdan for reading this note and making useful comments.

0. Norm. We shall now describe the algebra $E_{\varepsilon}^{\prime}=F_{\varepsilon} \otimes_{F} E$. Put $L=F_{\varepsilon} E, L^{\prime}=F_{\varepsilon}$ $\cap E$ and $l=\left[L^{\prime}: F\right]$. If $e=[E: F], n=\left[F_{\varepsilon}: F\right], e=e^{\prime} l$, and $n=n^{\prime} l$, then $e^{\prime}=$ $\left[E: L^{\prime}\right]=\left[L: F_{\varepsilon}\right]$ and $n^{\prime}=\left[F_{\varepsilon}: L^{\prime}\right]=[L: E]$. The galois group $\operatorname{Gal}(L / F)$ is generated by $\sigma$ and $\Phi$, where the restriction of $\sigma$ to $E$ generates $\operatorname{Gal}(E / F)=\mathbf{Z} / e \mathbf{Z}$; the restriction of $\Phi$ to $F_{\varepsilon}$ generates $\operatorname{Gal}\left(F_{\varepsilon} / F\right)=\mathbf{Z} / n \mathbf{Z} . F_{\varepsilon}$ (resp. $L^{\prime}$ ) is the fixed field of $\sigma^{\prime}$ in $L$ (resp. $E$ ). $E$ (resp. $L^{\prime}$ ) is the fixed field of $\Phi^{\prime}$ in $L$ (resp. $F_{\varepsilon}$ ).

The algebra $E_{\varepsilon}^{\prime}=F_{\varepsilon} \otimes_{F} E$ is a direct sum $L \oplus \cdots \oplus L$ of $l$ copies of $L$. It is viewed as a subalgebra of the $n \times n$ matrix algebra $M_{n}(E)$ over $E$ by fixing an embedding of $L$ in $M_{n^{\prime}}(E)$. Taking $\sigma^{l}$-invariants this yields an embedding of $F_{\varepsilon}$ in $M_{n^{\prime}}\left(L^{\prime}\right)$. The restriction of the determinant map $M_{n^{\prime}}(E) \rightarrow E$ to $L$ is the norm map $N_{L / E}$ from $L$ to $E$. The restriction to $F_{\varepsilon}$ is $N_{F_{\varepsilon} / L^{\prime}}$. The multiplicative group $E_{\varepsilon}^{\prime \times}$ of $E_{\varepsilon}^{\prime}$ is the group $T(E)$ of $E$-valued points on a torus $T$ of $\mathrm{GL}(n)$ defined over $F$. Further, $T(F)$ is $F_{\varepsilon}^{\times}$.

The restriction of the automorphism $\sigma$ of $M_{n}(E)$ to the subalgebra $E_{\varepsilon}^{\prime}=L \oplus$ $\cdots \oplus L$ has the form $\sigma \delta=\left(\sigma \delta_{2}, \sigma \delta_{3}, \ldots, \sigma \delta_{1}\right)$ if $\delta=\left(\delta_{1}, \ldots, \delta_{l}\right)$ lies in $E_{\varepsilon}^{\prime}$. We have $N_{E / F} E_{\varepsilon}^{\prime}=N_{L / F_{\varepsilon}} L$ since

$$
\delta \cdot \sigma \delta \cdot \cdots \cdot \sigma^{e-1} \delta=\delta^{\prime} \cdot \sigma^{\prime} \delta^{\prime} \cdot \cdots \cdot \sigma^{l\left(e^{\prime}-1\right)} \delta^{\prime},
$$

where

$$
\delta^{\prime}=\delta_{1} \cdot \sigma \delta_{2} \cdot \sigma^{2} \delta_{3} \cdot \cdots \cdot \sigma^{l-1} \delta_{l} \quad(\text { in } L) .
$$

If $\sigma \delta=\delta$, then $\delta=\left(\delta_{1}, \sigma^{-1} \delta_{1}, \ldots, \sigma^{1-l} \delta_{1}\right)$ with $\delta_{1}=\sigma^{\prime} \delta_{1}$ in $F_{\varepsilon}$. In general det $\delta=$ $\operatorname{det} \delta_{1} \cdot \cdots \cdot \operatorname{det} \delta_{l}=N_{L / E}\left(\delta_{1} \cdots \delta_{l}\right)$ lies in $E$. If $\delta=\sigma \delta$, then $\operatorname{det} \delta$ is $N_{F_{\varepsilon} / L^{\prime}}\left(N_{L^{\prime} / F} \delta_{1}\right)=N_{F_{\varepsilon} / F^{\prime}} \delta_{1}$. For transfer purposes we need

0.1 LEMMA. There exists a character $\eta=\left(\eta_{1}, \ldots, \eta_{l}\right)$ of $E_{\varepsilon}^{\prime \times}=L^{\times} \oplus \cdots \oplus L^{\times}$with $\varepsilon(\operatorname{det} \delta)=\eta(\sigma \delta / \delta), \delta$ in $E_{\varepsilon}^{\prime \times}$.

Proof. Since

$$
\varepsilon\left(\operatorname{det} \delta_{1} \cdots \cdots \operatorname{det} \delta_{l}\right)=\frac{\eta_{l}\left(\sigma \delta_{1}\right) \cdot \eta_{1}\left(\sigma \delta_{2}\right) \cdots \cdots \eta_{l-1}\left(\sigma \delta_{l}\right)}{\eta_{1}\left(\delta_{1}\right) \cdot \eta_{2}\left(\delta_{2}\right) \cdot \cdots \cdot \eta_{l}\left(\delta_{l}\right)}
$$

we have $\eta_{i}(\sigma x)=\eta_{i+1}(x) \varepsilon(\operatorname{det} x)$. Hence it suffices to find $\eta_{1}$ with

$$
\eta_{1}\left(\sigma^{l} x / x\right)=\varepsilon\left(N_{L / E}\left(x \sigma x \cdots \sigma^{l-1} x\right)\right) \quad\left(x \operatorname{in} L^{\times}\right) .
$$

But if $x=\sigma^{l} x$, then $x$ lies in $F_{\varepsilon}^{\times}$and the right side is $\varepsilon\left(N_{L^{\prime} / F} N_{F_{\varepsilon} / L^{\prime}} x\right)=1$.

1. Characters. We fix a character $\eta=\left(\eta_{1}, \ldots, \eta_{l}\right)$ of $T(E)=L^{\times} \oplus \cdots \oplus L^{\times}$as in Lemma 0.1. Then $\eta_{i+1}(x)=\eta_{i}(\sigma x) \varepsilon(\operatorname{det} x)^{-1}$ and $\eta_{1}\left(\sigma^{\prime} x / x\right)=\varepsilon^{\prime \prime}\left(N_{L / E} x\right)$ ( $x$ in $\left.L^{\times}\right)$, where $\varepsilon^{\prime \prime}(x)=\varepsilon\left(x \sigma x \cdots \sigma^{l-1} x\right)$ and $\sigma^{\prime}=\sigma^{l}$. Then there exists a one-to-one correspondence between the set of characters $\chi$ on $N_{L / F_{\mathrm{f}}} L^{\times}=N_{E / F} T(E)$, the set of characters $\chi_{1}$ of $L^{\times}=T_{1}(E)=\left(F_{\varepsilon} \otimes_{L^{\prime}} E\right)^{\times}$with ${ }^{\sigma^{\prime}} \chi_{1}=\chi_{1}{ }^{\sigma^{\prime}} \eta_{1} / \eta_{1}$ (it is given by 
$\left.\chi_{1}=\eta_{1} \cdot \chi \circ N_{L / F_{\mathrm{e}}}\right)$, and the set of characters $\chi_{E}=\left(\chi_{1}, \ldots, \chi_{l}\right)$ of $T(E)$ with ${ }^{\sigma} \chi_{E}=\chi_{E}{ }^{\sigma} \eta / \eta\left(=\chi_{E} \cdot \varepsilon \circ\right.$ det), where ${ }^{\sigma} \eta(\delta)=\eta(\sigma \delta)$ (it is given by $\chi_{E}=\eta$. $\chi \circ N_{E / F}$, which has the component

$$
\left.\chi_{i+1}={ }^{\sigma^{i}} \chi_{1} \eta_{i+1} /{ }^{\sigma^{i}} \eta_{1}={ }^{\sigma^{i}} \chi_{1}\left[\left(\varepsilon^{\sigma} \varepsilon \cdots \sigma^{\sigma^{i-1}} \varepsilon\right) \circ \operatorname{det}\right]^{-1}\right) \text {. }
$$

Namely we have a commutative diagram of character groups:

$$
\begin{array}{rll}
\hat{T}(F) \ni \chi & \mapsto \quad \chi_{1} \in \hat{T}_{1}(E) \\
& \searrow \quad \downarrow \\
& & \chi_{E} \in \hat{T}(E)
\end{array}
$$

These homomorphisms can be described also as $L$-group [B] homomorphisms ${ }^{L} T(F) \rightarrow{ }^{L} T_{1}(E),{ }^{L} T(F) \rightarrow{ }^{L} T(E),{ }^{L} T_{1}(E) \rightarrow{ }^{L} T(E)$. To be explicit, if $W_{L / F_{\varepsilon}}$ is the relative Weil group [T] of $L$ over $F_{\varepsilon}$, namely we have $1 \rightarrow C_{L} \rightarrow W_{L / F_{\varepsilon}} \rightarrow \operatorname{Gal}\left(L / F_{\varepsilon}\right)$

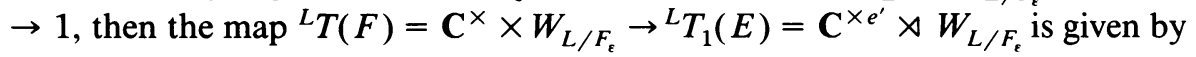

$$
\left(z ; u ; \sigma^{\prime i}\right) \mapsto\left(z \eta_{1}(u), z \eta_{1}\left(\sigma^{\prime} u\right), \ldots, z \eta_{1}\left(\sigma^{\prime e^{\prime}-1} u\right) ; u ; \sigma^{\prime i}\right) .
$$

Here $z$ in $\mathbf{C}^{\times}, u$ in $L^{\times}, 0 \leq i<e^{\prime}$, and $\sigma^{\prime}$ acts on $\mathbf{C}^{\times e^{\prime}}$ by permutation.

1.1. L-groups. The relations between the characters $\chi, \chi_{1}, \chi_{E}$ of $T(F)=F_{\varepsilon}^{\times}$, $T_{1}(E)=L^{\times}$and $T(E)=L^{\times} \oplus \cdots \oplus L^{\times}$are explained in $\S 1$. The lifting of these to representations $\tau_{1}$ of $\operatorname{GL}\left(n^{\prime}, E\right)$ with ${ }^{\sigma^{\prime}} \tau_{1} \cong \tau_{1} \otimes \varepsilon^{\prime \prime}$ and $\pi$ of $\operatorname{GL}(n, E)$ with ${ }^{\sigma} \pi \cong$ $\pi \otimes \varepsilon$ is compatible with an $L$-group homomorphism ${ }^{L} T(E) \rightarrow{ }^{L} \mathrm{GL}(n, E)$, which splits as $l$ copies of the map ${ }^{L} T_{1}(E) \rightarrow{ }^{L} \mathrm{GL}\left(n^{\prime}, E\right)$. We regard the groups $T_{1}(E)$, $T(E), \mathrm{GL}\left(n^{\prime}, E\right)$ and $\mathrm{GL}(n, E)$ as groups defined over $E$. They can be viewed as groups over $F$ on restricting scalars from $E$ to $F$. To define the map note that ${ }^{L} T_{1}(E)$ is the semidirect product of $\mathrm{C}^{\times n^{\prime}}$ and $\operatorname{Gal}(L / E)$ (which permutes the factors of $\left.\mathrm{C}^{\times n^{\prime}}\right)$, and ${ }^{L} \mathrm{GL}\left(n^{\prime}, E\right)$ is the direct product of $\mathrm{GL}\left(n^{\prime}, \mathbf{C}\right)$ and the galois group. We embed $\mathbf{C}^{\times n^{\prime}}$ as the diagonal in $\operatorname{GL}\left(n^{\prime}, \mathbf{C}\right)$, and map the generator $\Phi^{\prime}$ of $\operatorname{Gal}(L / E)$ to $w \times \Phi$. Here $w$ is the matrix of $\operatorname{GL}\left(n^{\prime}, \mathbf{C}\right)$ with $(i, j)$ entry 1 if $i+1 \equiv j\left(\bmod n^{\prime}\right)$, and 0 elsewhere. If $\tau_{1}$ is the image in ${ }^{L} \mathrm{GL}\left(n^{\prime}, E\right)$ of $\chi_{1}$ (in $\hat{T}_{1}(E)$ ), then $\chi_{E}$ (in $\hat{T}(E)$ ) maps to $\left(\tau_{1},{ }^{\sigma} \tau_{1} \otimes \varepsilon^{\prime}, \ldots\right)$.

2. Unramified case. Let $F$ be a local field, $E$ an unramified extension of $F$ of degree $e \geq 1, \varepsilon$ the unramified character of $E^{\times}$with $\varepsilon(\tilde{\omega})=\zeta, \zeta=e^{2 \pi i / n}$, where $\tilde{\omega}$ is a local uniformizer in $F$ (hence in $E$ ). For $x=u \tilde{\omega}^{r}(r$ in $\mathbf{Z}, u$ a unit in the ring $\mathcal{O}$ of integers of $F$ ) in $F^{\times}$we write $\operatorname{ord}(x)=r$ and $|x|=q^{-r}$, where $q$ is the number of elements in $\mathcal{O} / \tilde{\omega} \mathcal{O}$. The restriction of $\varepsilon$ to $F$ has order $n$. Since $\varepsilon$ is of order $n$ on $E^{\times}$it determines the unramified extension $E_{\varepsilon}$ of $E$ of degree $n$, by class field theory. The kernel of the character $\varepsilon(g)=\varepsilon(\operatorname{det} g)$ of $G(E)$ is denoted by $G_{0}(E)$. The quotient

$$
G(E) / G_{0}(E) \cong E^{\times} / N_{E_{\varepsilon} / E} E_{\varepsilon}^{\times} \cong \operatorname{Gal}\left(E_{\varepsilon} / E\right) \cong \mathbf{Z} / n \mathbf{Z}
$$

acts on the set $R\left(G_{0}\right)$ of equivalence classes of irreducible admissible representations $\rho$ of $G_{0}(E)$ by $g: \rho \rightarrow \rho^{g}$, where $\rho^{g}(h)=\rho\left(g^{-1} h g\right)$.

Denote by $R^{0}\left(G_{0}\right)$ the set of $\rho$ in $R\left(G_{0}\right)$ with $\rho^{g} \neq \rho$ for all $g \neq e$ in $G(E) / G_{0}(E)$. Signify by $K$ the maximal compact subgroup $G\left(\mathcal{O}_{E}\right)$ of $G(E)$. It is also a maximal compact subgroup in $G_{0}(E)$, and we denote by $R_{K}^{0}\left(G_{0}\right)$ the set of unramified 
elements in $R^{0}\left(G_{0}\right)$. Let $Z$ be the center of $G$. Then $Z(E)$ is the center of $G_{0}(E)$. Fix a character $\theta$ of $E^{\times} \cong Z(E)$, and let $R_{K}^{0, \theta}\left(G_{0}\right)$ be the $\rho$ in $R_{K}^{0}\left(G_{0}\right)$ with $\rho(z)=\theta(z) I$ for $z$ in $Z(E) . R_{K}^{0, \theta}\left(G_{0}\right)$ is empty unless $\theta$ is unramified, in which case $R_{K}^{0, \theta}\left(G_{0}\right)$ consists of a single element $\rho_{\theta}$ by [K, $\S 2$, Corollary, p. 219].

Recall $[\mathbf{K}, \S 2]$ that $\rho_{\theta}$ is the unique unramified irreducible component in the restriction of $\pi_{\theta}$ to $G_{0}(E)$, where $\pi_{\theta}$ is the unramified representation of $G(E)$ constructed as follows. For any $s=\left(s_{1}, \ldots, s_{n}\right)$ in $\mathbf{C}^{\times n}$ let $\chi_{s}$ be the unramified character of the upper triangular Borel subgroup $B(E)$ of $G(E)$ whose value at the diagonal matrix $\left(\tilde{\omega}^{r_{1}}, \ldots, \tilde{\omega}^{r_{n}}\right)$ is $s_{1}^{r_{1}} \cdots s_{n}^{r_{n}}$. The induced representation $\tilde{\pi}_{s}$ of $G(E)$ from $\chi_{s}$ on $B(E)$ contains a unique unramified irreducible constituent $\pi_{s}$. Now the unramified character $\theta$ of $E^{\times}$is determined by its value $\lambda=\theta(\tilde{\omega})$ at $\tilde{\omega}$, and $\lambda$ determines an $n$-tuple $s_{\mu}=\left(\mu, \zeta \mu, \ldots, \zeta^{n-1} \mu\right)$ in $\mathbf{C}^{\times n}$ up to permutation, by $\lambda=$ $\mu^{n} \xi^{n(n-1) / 2}$. Then $\pi_{\theta}=\pi_{s_{\mu}}$. It is clear that the unramified $\rho_{\theta}$ and $\pi_{\theta}$ are $\sigma$-invariant, where $\sigma$ is a generator of the cyclic galois $\operatorname{group} \operatorname{Gal}(E / F)$, namely, ${ }^{\sigma} \pi_{\theta} \cong \pi_{\theta}$, ${ }^{\sigma} \rho_{\theta} \cong \rho_{\theta}$.

3. Transfer. Let $\Phi$ be a generator of the cyclic galois group $\operatorname{Gal}\left(F_{\varepsilon} / F\right)$. For any $h$ in $F_{\varepsilon}^{\times}$put

$$
\tilde{\Delta}(h)=\prod_{1 \leq i<j \leq n}\left(1-\Phi^{i}(h) / \Phi^{j}(h)\right)
$$

and

$$
\Delta(h)=\left|\tilde{\Delta}(h)^{2}\right|^{1 / 2} \zeta^{n(n-1) \operatorname{val}(\tilde{\Delta}(h)) / 2} .
$$

Note that $\tilde{\Delta}(h)^{2}$ lies in $F$, and that $\zeta^{n / 2}=-1$. The set $F_{\varepsilon}^{\prime}$ of $h$ in $F_{\varepsilon}^{\times}$with $\Delta(h) \neq 0$ is called the regular set of $F_{\varepsilon}$.

As in $\S 0$, the cyclic extension $F_{\varepsilon}$ of $F$ of order $n$ is the splitting field of an elliptic torus $T$ of $G$ over $F$. The multiplicative group of $E_{\varepsilon}^{\prime}=F_{\varepsilon} \otimes_{F} E$ is isomorphic to $T(E)$, and we choose $T$ so that the group of units of the ring of integers in $E_{\varepsilon}^{\prime}$ is isomorphic to $T(E) \cap K$. In particular $F_{\varepsilon}^{\times}$is isomorphic to $T(F)$ and $\mathcal{O}_{F_{\varepsilon}}^{\times}$to $T(F) \cap K$, under this isomorphism. If $\delta$ lies in $T(E)\left(=\left(F_{\varepsilon} \otimes_{F} E\right)^{\times}\right)$we define the norm $h=N \delta=N_{E / F} \delta$ to be the element $\delta \sigma(\delta) \cdots \sigma^{e-1}(\delta)$ of $T(E)$. The galois group $\operatorname{Gal}(E / F)$ is generated by $\sigma$. It acts on $G(E)$.

For any smooth function $f$ on $G(E)$ with $f(z g)=\theta(z)^{-1} f(g)(z$ in $Z(E))$ which is compactly supported on $G(E) \bmod Z(E)$ put

$$
I_{h}(f)=\eta(\delta) \int_{P G(E)} \varepsilon(x) f\left(x^{-1} \delta \sigma x\right) d x .
$$

We write $P G(E)$ for $G(E) / Z(E)$, and note that $\varepsilon(z)=1$ for $z$ in $Z(E)$ and $\theta(z / \sigma z)=1$ since $\theta$ is unramified. Note that $I_{h}(f)$ depends only on the conjugacy class of $h=N \delta$ in $G(F)$. The character $\eta$ is defined by Lemma 0.1 . We assume it is unramified.

Denote by ${ }^{\sigma} R(G)$ (resp. ${ }^{\sigma} R\left(G_{0}\right)$ ) the set of $\sigma$-invariant $\rho$ in $R(G)$ (resp. $R\left(G_{0}\right)$ ). For such $\rho$ we have ${ }^{\sigma} \rho \cong \rho$, so that there exists an invertible operator $A$ from the space of ${ }^{\sigma} \rho$ to the space of $\rho$ with ${ }^{\sigma} \rho(g)=A^{-1} \rho(g) A$. We write $\operatorname{tr} \rho(f \times \sigma)$ for the 
trace of the operator $\rho(f \times \sigma)=\int_{P G(E)} f(g) \rho(g) A d g$. This operator is of trace class for the above $f$. We take $A$ with $A^{e}=I$; since $A^{e}$ intertwines $\rho$ with itself it is a scalar for irreducible $\rho$ by Schur's lemma. If $\rho$ is unramified, then ${ }^{\circ} \rho=\rho$ and $A$ is a scalar for irreducible $\rho$. We take $A=I$ in this case, and then $\operatorname{tr} \rho(f \times \sigma)=\operatorname{tr} \rho(f)$ for our $f$. The same comments apply to irreducible $\rho$ in ${ }^{\circ} R\left(G_{0}\right)$ and $f$ on $G_{0}(E)$ with the same properties.

4. Let $\mathbf{H}_{\theta}$ be the convolution algebra of $K$-bi-invariant compactly supported modulo $Z(E)$ functions $f$ on $G(E)$ with $f(z g)=\theta(z)^{-1} f(g)(z$ in $Z(E))$.

Proposition. For any $f$ in $\mathbf{H}_{\theta}$ and $\delta$ in $T(E)$ with regular $h=N \delta$ in $N T(E)=$ $N_{L / F_{e}} L^{\times}$we have $\Delta(h) I_{h}(f)=\operatorname{tr} \pi_{\theta}(f \times \sigma)$.

To prove this, we first note that since $\pi_{\theta}$ is unramified we have ${ }^{\sigma} \pi_{\theta}(g)=\pi_{\theta}(\sigma(g))$ $=\pi_{\theta}(g)$, hence $\operatorname{tr} \pi_{\theta}(f \times \sigma)$ is equal to $\operatorname{tr} \pi_{\theta}(f)$.

5. LemMA. If $f$ in $\mathbf{H}_{\theta}$ satisfies $\operatorname{tr} \pi_{\theta}(f)=0$, then $I_{h}(f)=0$ for all $h$.

Proof. Put $\pi$ for $\pi_{\theta}$. Since $\operatorname{tr} A B=\operatorname{tr} B A$ and there is an invertible operator $A$ on the space of $\pi$ with $\pi(g)=\varepsilon(g) A \pi(g) A^{-1}$, we have

$$
\operatorname{tr} \int f(g) \pi(g) d g=\operatorname{tr} \int \varepsilon(g) f(g) A \pi(g) A^{-1} d g=\operatorname{tr} \int \varepsilon(g) f(g) \pi(g) d g
$$

so that we may assume that $f$ is supported on $G_{0}(E)$. Note that $I_{h}(f)$ depends only on the restriction of $f$ to $G_{0}(E)$ (see Lemma 11 below). Choose $\beta$ in $G(E)$ with $\varepsilon(\beta)=\zeta$ and define the smooth function $\tilde{f}$ on $G_{0}(E)$ by

$$
\tilde{f}(g)=\sum_{i=0}^{n-1} \zeta^{-i} f\left(\beta^{-i} g \sigma \beta^{i}\right) .
$$

Explicitly $\beta$ can be taken to be the diagonal matrix $(\tilde{\omega}, 1, \ldots, 1)$ in $G(F)$.

5.1. We claim that for any $\rho$ in $R\left(G_{0}\right)$ we have $\operatorname{tr} \rho(\tilde{f} \times \sigma)=0$. Otherwise $\rho$ must transform under $Z(E)$ by $\theta$ and be $\sigma$-invariant, hence there is a nonzero intertwining operator $A$ with $\rho(g) A=A \rho(\sigma(g))$ and $A^{e}=1$. Now

$$
\begin{aligned}
\operatorname{tr} \int_{P G_{0}(E)} \tilde{f}(g) \rho(g) A d g & =\sum_{i=0}^{n-1} \zeta^{-i} \operatorname{tr} \int_{P G_{0}(E)} f\left(\beta^{-i} g \sigma \beta^{i}\right) \rho(g) A d g \\
& =\sum \zeta^{-i} \operatorname{tr} \int f(g) \rho^{\beta^{i}}(g) A d g=\sum \zeta^{-i} \operatorname{tr} \rho^{\beta^{i}}(f \times \sigma) .
\end{aligned}
$$

Here $P G_{0}(E)$ is $G_{0}(E) / Z(E)$. If $\rho^{\alpha} \cong \rho$ for some $\alpha=\beta^{i}(1 \leq i<n)$ then there exists an operator $B$ so that $\rho^{\alpha} B=B \rho$, and since $\sigma(\alpha)=\alpha, B$ can be assumed to commute with $A$. But then $\operatorname{tr} \rho(\tilde{f} \times \sigma)=0$. Moreover, if $\operatorname{tr} \rho^{\alpha}(f \times \sigma) \neq 0$, then $\rho^{\alpha}$ is unramified, hence lies in $R_{K}^{0, \theta}\left(G_{0}\right)$. This set consists of $\rho_{\theta}$ alone, hence

$$
\operatorname{tr} \rho(\tilde{f} \times \sigma)=\zeta^{-i} \operatorname{tr} \rho_{\theta}(f \times \sigma)=\zeta^{-i} \operatorname{tr} \pi_{\theta}(f \times \sigma) .
$$

This is 0 by the assumption of the lemma.

5.2. Kazhdan's completeness argument implies that since $\operatorname{tr} \rho(\tilde{f} \times \sigma)=0$ for all $\rho$ in $R\left(G_{0}\right)$, then

$$
I_{h}(f)=\eta(\delta) \int_{P G_{0}(E)} \tilde{f}\left(x^{-1} \delta \sigma(x)\right) d x
$$


is 0 for all $\delta$ with regular $h=N \delta$, as required. To sketch this argument we take a global field $F$ whose completion at $v_{0}$ is our local field, and a function $\tilde{f}=\otimes \tilde{f}_{v}$ on $P G_{0}\left(\mathbf{A}_{E}\right)$ whose component at $v_{0}$ is our local $\tilde{f}$, at a place $v_{1}$ which splits completely in $E / F$ it is a supercusp form, and at a place $v_{2}$ it is supported on the set of $\tilde{\delta}$ in $P G_{0}\left(E_{v_{2}}\right)$ with elliptic base-change norm in $P G_{0}\left(F_{v_{2}}\right)$. The assumption $\operatorname{tr} \rho(\tilde{f} \times \sigma)$ $=0$ implies the vanishing of the representation theoretic side of the DeligneKazhdan trace formula, hence the finite sum of twisted orbital integrals of $\tilde{f}$ on $P G_{0}\left(\mathbf{A}_{E}\right)$ is 0 . Given $\delta$ in $P G_{0}\left(E_{v_{2}}\right)$, it suffices to choose a global element $\tilde{\delta}$ in $P G_{0}(E)$ near $\delta$ in $P G_{0}\left(E_{v_{2}}\right)$, and $\tilde{f}$ with $\int \tilde{f}\left(x^{-1} \tilde{\delta} \sigma(x)\right) d x \neq 0$ but $\int \tilde{f}\left(x^{-1} \tilde{\delta}^{\prime} \sigma(x)\right) d x$ $=0$ for $\tilde{\delta}^{\prime}$ in $P G_{0}(E)$ not $\sigma$-conjugate to $\tilde{\delta}$, to deduce a contradiction to the assumption $\int \tilde{f}_{v_{0}}\left(x^{-1} \delta \sigma(x)\right) d x \neq 0$.

6. It follows from Lemma 5 that there exists a function $\varphi$ on the set of regular $h=N \delta$ in $N T(E)$ so that

$$
I_{h}(f)=\varphi(h) \operatorname{tr} \pi_{\theta}(f \times \sigma)
$$

for all $f$ in $\mathbf{H}_{\theta}(G)$ and the above $h$.

Define $\varphi_{\theta}$ in $\mathbf{H}_{\theta}$ by $\varphi_{\theta}(g)=0$ unless $g=z k$ ( $z$ in $Z(E), k$ in $K$ ), where $\varphi_{\theta}(g)=\theta^{-1}(z)|K|^{-1}$. We denote by $|K|$ the volume of $K$. Since $\operatorname{tr} \pi_{\theta}\left(\varphi_{\theta} \times \sigma\right)=1$ we have

$$
\varphi(h)=\eta(\delta) \int_{\substack{x \in P G(E) \\ x^{-1} \delta \sigma(x) \in K}}|K|^{-1} \varepsilon(x) d x
$$

for all $\delta$ with regular $h=N \delta$. We may assume that $\delta$ lies in $T(E) \cap K$, in which case $\eta(\delta)=1$.

6.1. LemMA. For any $\delta$ inT $(E) \cap K$ with regular $h=N \delta$ we have $\varphi(h)=\Delta(h)^{-1}$.

Proof. In the case $E=F$ this is Kazhdan's lemma [K, $\S 3$, Theorem 1]. To reduce the general case to this case we use Kottwitz's base-change lemma [Ko], which we now recall. We have to study

$$
\int_{\left\{g \in P G(E) \mid g^{-1} \delta \sigma(g) \in K\right\}}|K|^{-1} \varepsilon(g) d g=\sum_{\substack{g \in G(E) / K \\ g^{-1} \delta \sigma(g) \in K}} \varepsilon(g)=\sum_{x \in X_{E}^{\delta \sigma}} \varepsilon(x) .
$$

The last sum is over all vertices $x=g x_{0}$ in the Bruhat-Tits building $X_{E}$ [Ti] of $G(E)$ which are fixed by $\delta \sigma$, namely $\delta \sigma x=x$. Here $x_{0}$ is the hyperspecial point in the building $X_{F}$ of $G(F)$ whose stabilizer in $G(F)$ is $G(\mathcal{O})=K(F)$, and $K=G\left(\mathcal{O}_{E}\right)$ in $G(E)$. We put $\varepsilon(x)=\varepsilon(g)$. This has to be compared with

$$
\sum_{x \in X_{F}^{h}} \varepsilon(x)=\int_{\left\{g \in P G(F) ; g^{-1} h g \in K(F)\right\}}|K(F)|^{-1} \varepsilon(g) d g,
$$

where $h=N \delta=\delta \sigma(\delta) \cdots \sigma^{e-1}(\delta)(e=[E: F])$ is an element of $T(F) \cap K$ since $\delta \sigma(\delta)=\sigma(\delta) \delta$. Let $\bar{F}$ be the completion of the maximal unramified extension $F^{\text {ur }}$ of 
$F$. Denote by $\Phi$ a Frobenius element in $\operatorname{Gal}(\bar{F} / F)$ whose restriction to $E$ is our $\sigma$. We have

$$
X_{\bar{F}}=G(\bar{F}) x_{0} \supset X_{E}=G(E) x_{0} \supset X_{F}=G(F) x_{0} .
$$

The semidirect product $G(\bar{F}) \rtimes\langle\Phi\rangle$ acts on the building $X_{\bar{F}}$, and

$$
X_{\bar{F}}^{\langle h, \Phi\rangle}=X_{F}^{h}, \quad X_{\bar{F}}^{\left\langle\delta \Phi, \Phi^{e}\right\rangle}=X_{E}^{\delta \sigma} .
$$

If $b=1$ and $a=e-1$, then the determinant of $\left(\begin{array}{cc}b & a \\ 1 & e\end{array}\right)$ is 1 , so that the group $\langle h, \Phi\rangle$ is equal to $\left\langle h^{a} \Phi^{e}, h \Phi\right\rangle$. Since the map from $G\left(\mathcal{O}_{\bar{F}}\right)$ to itself by $g \mapsto g^{-1} \Phi^{e}(g)$ is surjective, given our $h$ in $T(F) \cap K$ there exists $c$ in $G\left(\mathcal{O}_{\bar{F}}\right)$ with $h^{a}=c^{-1} \Phi^{e}(c)$. Put $\delta^{\prime}=\operatorname{ch} \Phi(c)^{-1}$ (in $G\left(\mathcal{O}_{\bar{F}}\right)$ ) to obtain that $\left\langle h^{a} \Phi^{e}, h \Phi\right\rangle=c^{-1}\left\langle\Phi^{e}, \delta^{\prime} \Phi\right\rangle c$ and $X_{F}^{h}=$ $c^{-1} X_{E}^{\delta^{\prime} \sigma}$. Since $\langle h, \Phi\rangle$ is commutative, $\delta^{\prime}=\Phi^{e}\left(\delta^{\prime}\right)$ lies in $G(E)$, in fact in $K$. As $N \delta^{\prime}=c h c^{-1}$ and $N \delta=h$ are conjugate, and $\delta, \delta^{\prime}$ lie in $K$, there exists $c_{0}$ in $K$ with $\delta^{\prime}=c_{0} \delta \sigma\left(c_{0}\right)^{-1}$. Then $X_{F}^{h}=c^{-1} c_{0} X_{F}^{\delta \sigma}$, and since $\varepsilon\left(c_{0} c^{-1}\right)=1$ we have

$$
\sum_{x \in X_{E}^{\delta o}} \varepsilon(x)=\sum_{x \in X_{F}^{h}} \varepsilon(x) .
$$

The right side here is equal to $\Delta(h)^{-1}$ by Kazhdan's lemma.

This terminates the proof of Lemma 6.1, hence Proposition 4.

7. Reformulation. The groups $T(E)$ and $G(E)$ can be viewed as groups over $F$ upon restricting scalars from $E$ to $F$, and we may take the form of the $L$-group with respect to the galois group $\operatorname{Gal}(\bar{F} / F)$ of the unramified closure $\bar{F}$ of $F$; thus ${ }^{L} H={ }^{L} H^{0} \rtimes \operatorname{Gal}(\bar{F} / F)$, where $H(F)=T(F), T(E)$ or $G(E)$. The homomorphisms of $\$ \S 1$ and 1.1 define $L$-group homomorphisms ${ }^{L} T(F) \rightarrow{ }^{L} T(E) \rightarrow{ }^{L} G(E)$, whose composition we denote by $c$. There is a canonical isomorphism [C] between semisimple conjugacy classes $s$ in ${ }^{L} H$ whose projection on $\operatorname{Gal}(\bar{F} / F)$ is the Frobenius element $\Phi$, and irreducible unramified representations $\pi_{s}$ of $H(F)$. For any spherical function $f$ in the Hecke algebra $\mathbf{H}=\mathbf{H}(H(F), H(\mathcal{O}))$ we denote by $f^{\vee}$ the function on ${ }^{L} H^{0} \times \Phi$ given by $f^{\vee}(s)=\operatorname{tr} \pi_{s}(f \times \Phi)$. The Satake map is an isomorphism between $\mathbf{H}$ and the algebra of regular class functions on ${ }^{L} H$.

Now put $\mathbf{H}_{G}=\mathbf{H}\left(G(E), G\left(\mathcal{O}_{E}\right)\right)$ and $\mathbf{H}_{T}=\mathbf{H}(T(F), T(\mathcal{O}))$. The map $c^{*}: \mathbf{H}_{G} \rightarrow$ $\mathbf{H}_{T}$ dual to $c:{ }^{L} T(F) \rightarrow{ }^{L} G(E)$ is defined by $\left(c^{*} f\right)^{\vee}(s)=f^{\vee}(c(s))$, namely, $\left(c^{*} f\right)^{\vee}$ is the restriction of $f^{\vee}$ to ${ }^{L} T(F)$.

7.1. Proposition. For any $f$ in $\mathbf{H}_{G}$ and $h$ in $N T(E)$ we have $\Delta(h) I_{h}(f)=\left(c^{*} f\right)(h)$.

Proof. This is equivalent to Proposition 4. Indeed

$$
\operatorname{tr} \pi_{\theta}(f \times \Phi)=f^{\vee}(c(\theta))=\left(c^{*} f\right)^{\vee}(\theta)=\theta\left(c^{*} f\right)=\left(c^{*} f\right)(h) .
$$

8. $F_{\varepsilon}$ splits. Let $m$ be a divisor of $n, F_{m}$ the unramified extension of $F$ of degree $m$, and $n^{\prime}=n / m$. Suppose $F_{\varepsilon}$ splits as a direct sum of $n^{\prime}$ copies of $F_{m}$. Then $T(F)=\oplus T_{1}(F)$ with $T_{1}(F) \cong F_{m}^{\times} \subset \mathrm{GL}(m, F)$, so that $T(F)$ lies in the Levi component $M(F)=\mathrm{GL}(m, F)^{n^{\prime}}$ of a standard parabolic subgroup $P(F)$ of $G(F)$ $=\mathrm{GL}(n, F)$. The map

$$
c:{ }^{L} T(F) \rightarrow{ }^{L} T(E) \rightarrow{ }^{L} G(E)
$$


splits through $c=j \circ \oplus c_{i}$, where $j$ is the embedding ${ }^{L} M(E) \rightarrow{ }^{L} G(E)$ and $c_{i}$ : ${ }^{L} T_{1}(F) \rightarrow{ }^{L} T_{1}(E) \rightarrow{ }^{L} \mathrm{GL}(m, E)$. Note that $j^{*} f=f_{N}^{K}$, where $N$ is the unipotent radical of $P$ and $K=\mathrm{GL}\left(n, \mathcal{O}_{E}\right)$, and for $m$ in $M(E)$ we put

$$
\begin{aligned}
f_{N}^{K}(m) & =\int_{K} \int_{N(E)} f\left(k^{-1} m n \sigma(k)\right) d n d k \\
& =|\operatorname{det}(\operatorname{Ad}(N m)-I)|_{\text {Lie } N(F)} \mid \iint f\left(k^{-1} n^{-1} m \sigma(n k)\right) d n d k .
\end{aligned}
$$

Here $N m=N_{E / F} m$ can be viewed as an element of $M(F)$, and $\operatorname{Ad} h$ indicates the adjoint action of $h$ on the Lie algebra of $N(F) . j^{*}$ is the map dual to $j$ from $\mathbf{H}_{G}=\mathbf{H}\left(G(E), G\left(\mathcal{O}_{E}\right)\right)$ to $\mathbf{H}\left(M(E), M\left(\mathcal{O}_{E}\right)\right)$.

For $\delta$ in $T(E) \subset M(E)$ and $h=N \delta$ in $T(F)$ define

$$
D(h)=\operatorname{det}\left[\left.(\operatorname{Ad}(h)-I)\right|_{\text {Lie } G(F) / \operatorname{Lie} G_{h}(F)}\right],
$$

where $G_{h}\left(\right.$ resp. $\left.M_{h}\right)$ is the centralizer of $h$ in $G($ resp. $M)$. Note that $M_{h}=G_{h}$. Also put

$$
D^{\prime}(h)=\operatorname{det}\left[\left.(\operatorname{Ad}(h)-I)\right|_{\text {Lie } M(F) / \text { Lie } M_{h}(F)}\right]
$$

Define

$$
\Delta(h)=|\tilde{\Delta}(h)| \zeta_{m}^{m(m-1) \operatorname{val}(\tilde{\Delta}(h)) / 2}=|\tilde{\Delta}(h)| \zeta_{m}^{m(m-1) \operatorname{val}\left(\tilde{\Delta}^{\prime}(h)\right) / 2},
$$

where $\zeta_{m}=\exp (2 \pi i / m)$ and $\tilde{\Delta}(h), \tilde{\Delta}^{\prime}(h)$ are elements of $F$ whose squares have valuations equal to those of $D(h), D^{\prime}(h)$, respectively. Note that $D^{\prime}(h)$ and $\tilde{\Delta}^{\prime}(h)$ split naturally as products of $n^{\prime}$ factors $D_{i}^{\prime}(h)$ and $\tilde{\Delta}_{i}^{\prime}(h)$. Also put

$$
I_{h}(f)=\eta(\delta) \int_{G_{\delta}^{\sigma}(E) / G(E)} \varepsilon_{m}(x) f\left(x^{-1} \delta \sigma(x)\right) d x,
$$

where $\varepsilon_{m}(x)=\zeta_{m}^{\text {val(det } x)}$, and set $f_{\varepsilon}=c^{*} f$, where $c^{*}: \mathbf{H}_{G} \rightarrow \mathbf{H}_{T}$ is the map dual to $c$ : ${ }^{L} T(F) \rightarrow{ }^{L} G(E)$. Note that $\eta$ is a product of $n^{\prime}$ characters.

8.1. LeMMA. For a regular $h$ in $N T(E)$ we have $\Delta(h) I_{h}(f)=f_{\varepsilon}(h)$.

Proof. $j^{*} f$ is a linear combination of products $\prod_{i=1}^{n^{\prime}} f_{i}$ of functions $f_{i}$ in $\mathbf{H}\left(\mathrm{GL}(m, E), \mathrm{GL}\left(m, \mathcal{O}_{E}\right)\right)$. Thus $\Delta(h) I_{h}(f)$ is a linear combination of products of

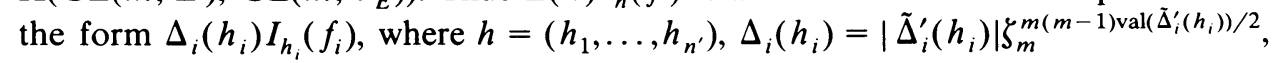
and $I_{h_{i}}$ is defined using $\eta_{i}$ and an integral over $G_{\delta_{i}}^{\prime \sigma}(E) \backslash G^{\prime}(E)$, where $G^{\prime}=\operatorname{GL}(m)$. But Proposition 7.1 implies that $\Delta_{i}\left(h_{i}\right) I_{h_{i}}\left(f_{i}\right)=\left(c_{i}^{*} f_{i}\right)\left(h_{i}\right)$. Since $c^{*} f$ is a linear combination of products over $i$ of $c_{i}^{*} f_{i}$, the lemma follows.

9. $E$ splits. We shall now discuss the case where $E$ splits as a direct sum $R \oplus \cdots \oplus R$ of $e^{\prime}$ copies of a field extension $R$ of degree $r$ over $F$. Then $G(E)=G(R) \oplus \cdots \oplus G(R)$ and $\operatorname{Gal}(E / F)$, which is generated by $\sigma$, acts by

$$
\sigma x=\left(x_{2}, x_{3}, \ldots, x_{e^{\prime}}, x_{1}\right), \text { where } x=\left(x_{1}, x_{2}, \ldots, x_{e^{\prime}}\right) .
$$

Then $\sigma^{e^{\prime}} x=\tau x$ generates $\operatorname{Gal}(R / F)$. The function $f=\left(f_{1}, \ldots, f_{e^{\prime}}\right)$ is an $e^{\prime}$-tuple, where $f_{2}=\cdots=f_{c^{\prime}}$ is the characteristic function of the maximal compact subgroup 
$G\left(\mathcal{O}_{R}\right)$, and $f_{1}$ is any spherical function in $\mathbf{H}_{R}$. Also $\varepsilon(x)=\varepsilon\left(x_{1} \ldots x_{e^{\prime}}\right)$, where $\varepsilon\left(x_{1}\right)=\zeta_{n}^{\operatorname{val}\left(\operatorname{det} x_{1}\right)}, \zeta_{n}=e^{2 \pi i / n}$. We take $\delta=\left(\delta_{1}, \ldots, \delta_{e^{\prime}}\right)$ with $\delta_{i}$ in $G\left(\mathcal{O}_{R}\right)$, so that

$$
\begin{aligned}
\int_{P G(E)} \varepsilon(x) f\left(x^{-1} \delta \boldsymbol{\sigma}(x)\right) d x & \\
& =\int \varepsilon\left(x_{1} \ldots x_{e^{\prime}}\right) f_{1}\left(x_{1}^{-1} \delta_{1} x_{2}\right) f_{2}\left(x_{2}^{-1} \delta_{2} x_{3}\right) \cdots f_{e^{\prime}}\left(x_{e^{\prime}}^{-1} \delta_{e^{\prime}} \tau x_{1}\right) .
\end{aligned}
$$

Here

$$
x_{e^{\prime}}=\delta_{e^{\prime}} \tau x_{1}, x_{e^{\prime}-1}=\delta_{e^{\prime}-1} x_{e^{\prime}}, \ldots, x_{2}=\delta_{2} x_{3}=\delta_{2} \delta_{3} \cdots \delta_{e^{\prime}-1} \delta_{e^{\prime}} \tau x_{1} .
$$

Since all $\delta_{i}$ lie in $G\left(\mathcal{O}_{R}\right)$ we obtain

$$
\int_{P G(R)} \varepsilon\left(x_{1}\right)^{e^{\prime}} f_{1}\left(x_{1}^{-1} \delta_{1} \cdots \delta_{e^{\prime}} \tau x_{1}\right) d x_{1}=\int_{P G(R)} \varepsilon_{r}\left(x_{1}\right) f_{1}\left(x_{1}^{-1} \delta_{0} \tau x_{1}\right) d x_{1} .
$$

Here $\delta_{0}=\delta_{1} \cdots \delta_{e^{\prime}}$ lies in $G\left(\mathcal{O}_{R}\right)$ and we require that $N_{R / F} \delta_{0}$ is regular in $G(F)$. Also $\varepsilon_{r}\left(x_{1}\right)=\zeta_{r}^{\left.\text {val(det } x_{1}\right)}$, where $\zeta_{r}=e^{2 \pi i / r}, r=[R: F]$. Proposition 7.1 and Lemma 8.1 now apply to $R, f_{1} \in \mathbf{H}_{R}$ in place of $E, f \in \mathbf{H}_{E}$.

10. Automorphic forms. Let $L^{2}=L^{2}\left(G(E) \backslash G\left(\mathbf{A}_{E}\right)\right)$ be the space of squareintegrable functions $\psi$ on $Z\left(\mathbf{A}_{E}\right) G(E) \backslash G\left(\mathbf{A}_{E}\right)$ which transform under $Z\left(\mathbf{A}_{E}\right)$ by $\theta$. Here $\theta$ is a character of $Z(E) \backslash Z\left(\mathbf{A}_{E}\right)$ with $\theta(\sigma z / z)=\varepsilon\left(z^{n}\right)$, where $\varepsilon$ is a character on $E^{\times} \backslash \mathbf{A}_{E}^{\times}$whose restriction to $\mathbf{A}^{\times}$is of order exactly $n$. Thus $\psi(z g)=\theta(z) \psi(g)$ for $z$ in $Z\left(\mathbf{A}_{E}\right)$ and $g$ in $G\left(\mathbf{A}_{E}\right)$. Let $f$ be a compactly supported (modulo $Z\left(\mathbf{A}_{E}\right)$ ) smooth function on $G\left(\mathbf{A}_{E}\right)$ which transforms under $Z\left(\mathbf{A}_{E}\right)$ by $\theta^{-1} \cdot G\left(\mathbf{A}_{E}\right)$ acts on $L^{2}$ by right translations: $r(g) \psi(h)=\psi(h g)$, and $L^{2}$ splits as a direct sum of invariant subspaces which appear discretely or continuously. Define the operator $r(\sigma \varepsilon) \psi(g)$ $=\varepsilon\left(\sigma^{-1} g\right) \psi\left(\sigma^{-1} g\right)$ on $L^{2}$, and consider the operator $r(f \sigma \varepsilon)$ which maps $\psi$ to the function (of $h$ ) in $L^{2}$ given by

$$
\begin{aligned}
r(f \sigma \varepsilon) \psi(h) & =\int_{Z\left(\mathbf{A}_{E}\right) \backslash G\left(\mathbf{A}_{E}\right)} f(\sigma g) r(g \sigma \varepsilon) \psi(h) d g \\
& =\int_{Z\left(\mathbf{A}_{E}\right) \backslash G\left(\mathbf{A}_{E}\right)} f(\sigma g) \varepsilon\left(\sigma^{-1}(h) g\right) \psi\left(\sigma^{-1}(h) g\right) d g \\
& =\sum_{\delta \in Z(E) \backslash G(E)} \int_{Z\left(\mathbf{A}_{E}\right) G(E) \backslash G\left(\mathbf{A}_{E}\right)} f\left(h^{-1} \boldsymbol{\delta} \sigma(g)\right) \varepsilon(g) \psi(g) d g .
\end{aligned}
$$

Arthur [A] proved an identity of the form $\Sigma_{\mathcal{O}} J_{\mathscr{O}}^{T}(f)=\Sigma_{\chi} J_{\chi}^{T}(f)$, generalizing Selberg's trace formula. In our case $\sigma$ and $\varepsilon$ preserve each parabolic subgroup $P$ of $G=\mathrm{GL}(n)$. Hence although [A] deals with the nontwisted situation only, the techniques generalize at once to deal with our situation. Let $V$ be a sufficiently large finite set of places.

10.1. Lemma. Suppose that $f=\otimes f_{v}$, where $f_{v}$ is spherical in $\mathbf{H}_{E}$ for $v$ outside $V$, and $f_{v}$ is supported on the o-regular set of $G\left(E_{v}\right)$ for $v$ in $V$, namely on the $\delta$ with $h=N_{E / F} \delta$ regular in $G\left(F_{v}\right)$. Then $\sum_{\mathcal{O}} J_{\mathscr{O}}^{T}(f)$ of $[\mathbf{A}]$ takes the form

$$
\sum_{\{\boldsymbol{\delta}\}} c(\boldsymbol{\delta}) \int_{Z\left(\mathbf{A}_{E}\right) G_{\delta}^{\sigma}(\mathbf{A}) \backslash G\left(\mathbf{A}_{E}\right)} \varepsilon(g) f\left(g^{-1} \boldsymbol{\delta} \boldsymbol{\sigma}(g)\right) v^{T}(g) d g,
$$


where the sum ranges over a set of representatives $\delta$ for the $\sigma$-conjugacy classes in $G(E) / Z(E)$ so that $N \delta$ is regular. $c(\delta)$ is a volume factor which is equal to $\left|G_{\delta}^{\sigma}(F) Z(\mathbf{A}) \backslash G_{\delta}^{\sigma}(\mathbf{A})\right|$ if $N \delta$ is elliptic and nonconjugate to $z N \delta$ for any $z$ in $Z(F)$. The weight factor $v^{T}(g)$ is left-invariant under $G_{\delta}^{\sigma}(\mathbf{A})$, a group isomorphic over $F$ to $G_{h}(\mathbf{A})$ if $h=N \delta$ lies in $G(F) \cdot v^{T}(g)$ equals 1 if $N \delta$ is elliptic regular.

Proof. The proof is clear from [A, Theorem 8.1], the definition of $J_{P, O}(x, y)$, and the computations on pp. 950-951 of [A].

Note that our assumption on $f_{v}(v$ in $V)$ is very restrictive. It would not permit deducing local results, for example, in the case of the Deligne-Kazhdan comparison of representations of $\mathrm{GL}(n)$ and the multiplicative groups of simple algebras. Since we compare here $\mathrm{GL}(n)$ and $\mathrm{GL}(1)$, the global strong multiplicity one theorem compensates (see Proposition 15.2) for the lack of local knowledge of the germs of $f_{\varepsilon}$.

11. LEMMA. The sum ranges only over $\delta$ with $N \delta$ conjugate in $G(E)$ to an elliptic (regular) element $h$ in a torus $T(F)$ of $G(F)$ whose splitting field is $F_{\varepsilon}$.

Proof. The integral vanishes unless $\varepsilon(g)=1$ for all $g$ in $G_{h}(\mathbf{A})$. Since $h$ is regular, $G_{h}(F)$ is isomorphic to a product $\prod_{i=1}^{r} L_{i}^{\times}$of the multiplicative groups of fields $L_{i}$ with $\sum_{i=1}^{r}\left[L_{i}: F\right]=n$, and $\varepsilon\left(\prod_{i=1}^{r} l_{i}\right)=\varepsilon\left(\prod_{i=1}^{r} N_{i}\left(l_{i}\right)\right), N_{i}$ is the norm map from $L_{i}$ to $F$. We have $N_{i} \mathbf{A}_{L_{i}}^{\times} \subset \operatorname{ker}\left(\varepsilon \mid \mathbf{A}^{\times}\right)=N_{F_{\varepsilon} / F} \mathbf{A}_{F_{\varepsilon}}^{\times}$for $1 \leq i \leq r$, since $\varepsilon(g)=1$ for $g$ in $G_{h}(\mathbf{A})$. Hence $L_{i}$ contains $F_{\varepsilon}$ for all $i$ so that $r=1$ and $L_{1}=F_{\varepsilon}$, as required.

12. $\chi$-expansion. We have to describe also the sum $\Sigma_{\chi} J_{\chi}^{T}(f)$ of $\left[\mathbf{A}^{\prime}\right]$. Fortunately most terms here vanish. It is a sum over

(1) all standard (containing a fixed minimal such $M_{0}$ ) Levi factors $M$ of parabolic subgroups $P$ of $G$ (defined over $E$ ),

(2) subspaces $\mathfrak{A}=\operatorname{Hom}\left(X\left(M_{R}\right), \mathbf{R}\right)$ of $\mathfrak{A}_{M}=\operatorname{Hom}(X(M), \mathbf{R})$,

(3) elements $s$ in the Weyl group $W^{\mathfrak{A}}\left(\mathfrak{A}_{M}\right)$ of distinct maps from $\mathfrak{A}_{M}$ to itself obtained by restriction of the elements of $W\left(\mathfrak{U}_{0}\right)$, the Weyl group of $\mathfrak{A}_{0}=\mathfrak{U}_{\mathcal{M}_{0}}$, such that their space of fixed vectors is precisely $\mathfrak{A}$,

(4) unitary discrete series representations $\tau$ of $M\left(\mathbf{A}_{E}\right)$ so that $s\left({ }^{\circ} \tau \otimes \varepsilon^{\prime}\right) \cong \tau$. We put $\varepsilon^{\prime}$ for $\varepsilon^{-1}$.

We have $M=\mathrm{GL}\left(n_{1}\right) \times \cdots \times \mathrm{GL}\left(n_{r}\right)$ and $n_{1}+\cdots+n_{r}=n, \tau=\left(\tau_{1}, \ldots, \tau_{r}\right)$ and $s$ permutes the factors. An orbit $\left(\tau^{\prime},{ }^{\sigma} \tau^{\prime} \otimes \varepsilon^{\prime},{ }^{\sigma^{2}} \tau^{\prime} \otimes \varepsilon^{\prime \sigma} \varepsilon^{\prime}, \ldots\right)$ of length $r^{\prime}$ in $\tau$ must be the entire $\tau$ since the restriction of $\varepsilon$ to $\mathbf{A}^{\times}$is of order $n$, and ${ }^{\sigma^{\prime}} \tau^{\prime} \otimes \varepsilon^{\prime \sigma} \varepsilon^{\prime}$ $\ldots \sigma^{r^{\prime}-1} \varepsilon^{\prime} \cong \tau^{\prime}$ implies that $\varepsilon^{\prime r^{\prime} n_{1}}=1$ on $\mathrm{A}^{\times}$. Hence $s$ does not have any fixed vectors except $\{0\}$, so that $\mathfrak{A}=\{0\}$, and $M$ is $\operatorname{GL}\left(n^{\prime}\right)^{r}$ with $r n^{\prime}=n$.

Hence $\sum_{\chi} J_{\chi}^{T}(f)$ is equal to the sum over $M=\mathrm{GL}\left(n^{\prime}\right)^{r}, n=r n^{\prime}$, and $s$ in $W^{\{0\}}\left(\mathfrak{A}_{M}\right)$, and discrete series representations $\tau$ of $M\left(\mathbf{A}_{E}\right)$ with $s\left({ }^{\sigma} \tau \otimes \varepsilon^{\prime}\right) \cong \tau$, of

$$
\left.\frac{\left[W_{0}^{M}\right]}{\left[W_{0}\right]}\left|\operatorname{det}\left(s \sigma \varepsilon^{\prime}-1\right)\right|_{\mathscr{A}_{M}}\right|^{-1} \operatorname{tr}[M(s) I(\tau, f \times \sigma \varepsilon)],
$$

where $W_{0}^{M}=W\left(M, M_{0}\right), W_{0}=W\left(G, M_{0}\right)$ and $M(s)$ is an operator intertwining the induced representation $I(\tau)=I_{M}^{G}(\tau)$ of $G$ from $\tau$ on $M$ with itself. As $I(\tau)$ is irreducible, $M(s)$ is a scalar. Hence we can write (12.1) in the form $c_{M} \operatorname{tr} I(\tau, f \times \sigma \varepsilon)$ with a constant $c_{M}$. 
13. O-expansion. The $\operatorname{sum} \sum_{\mathcal{O}} J_{\mathcal{O}}^{T}(f)$ takes the form

$$
\sum_{\delta}|Z(\mathbf{A}) T(F) \backslash T(\mathbf{A})| \int_{Z\left(\mathbf{A}_{E}\right) G_{\delta}^{o}(\mathbf{A}) \backslash G\left(\mathbf{A}_{E}\right)} \varepsilon(g) f\left(g^{-1} \boldsymbol{\delta} \sigma(g)\right) d g .
$$

As in $\S \S 0$ and $11, T$ is a fixed $F$-torus of $G$ whose splitting field is $F_{\varepsilon}$, so that $T(F)=F_{\varepsilon}^{\times}$. The sum ranges over $\sigma$-conjugacy classes in $G(E)$ of $\delta$ in $T(E) / Z(E)$ with regular norm $h=N \delta$, equivalently over conjugacy classes in $G(F)$ of regular $h$ in $T(F) / Z(F)$. This can be expressed as a sum over the regular $h$ in $T(F) / Z(F)$, divided by $n$. Indeed, $h$ and $h^{\prime}$ of $T(F) / Z(F)$ are conjugate in $G(F)$ if and only if $h^{\prime}=\tau h$ for some $\tau$ in $\operatorname{Gal}\left(F_{\varepsilon} / F\right)$. Since the Tamagawa number $|Z(\mathbf{A}) T(F) \backslash T(\mathbf{A})|$ and the cardinality of $\operatorname{Gal}\left(F_{\varepsilon} / F\right)$ are both $n$ we obtain

$$
\sum_{h \in N T(E) / N Z(E)} \int_{Z\left(\mathbf{A}_{E}\right) G_{\delta}^{\sigma}(\mathbf{A}) / G\left(\mathbf{A}_{E}\right)} \varepsilon(g) f\left(g^{-1} \delta \sigma g\right) d g,
$$

where by $N T(E)$ we mean $N_{E / F}\left(T(F) \otimes_{F} E\right)\left(=N_{L / F_{e}} L^{\times}\right.$, see $\left.\S 0\right)$, and $\delta$ is an element of $T(E)$ with regular $h=N \delta$.

For any $h$ in $F_{\varepsilon}^{\times} \cong T(F)$ put $\tilde{\Delta}(h)=\Pi\left(\Phi^{j}(h)-\Phi^{i}(h)\right)(1 \leq i<j \leq n)$. It satisfies $\Phi(\tilde{\Delta}(h))=(-1)^{n(n-1) / 2} \tilde{\Delta}(h)$. Fix $h_{0}$ in $F_{\varepsilon}^{\times}$with $\tilde{\Delta}\left(h_{0}\right) \neq 0$. Then $\tilde{\Delta}(h) \tilde{\Delta}\left(h_{0}\right)$ lies in $F$. Define $\Delta=\Delta_{h_{0}}$ by

$$
\Delta(h)=|N h|^{(1-n) / 2}|\tilde{\Delta}(h)| \varepsilon\left(\tilde{\Delta}(h) \tilde{\Delta}\left(h_{0}\right)\right)^{n(n-1) / 2} .
$$

This definition is regarded as a local definition, at each place $v$ of $F$, and as a global definition, for each idèle $h$ of $F_{\varepsilon}^{\times}$. In fact we fix a global $h_{0}$ in $F_{\varepsilon}^{\times}$, and note that for each global $h$ in $F_{\varepsilon}^{\times}$we have $\Delta(h)=1$. Since $h_{0}$ is global (rational), it is a unit at almost all $v$, and our definition coincides with the one given in $\S 3$ of $\Delta(h)$.

For any $\delta$ in $T\left(E_{v}\right)$ with regular $h=N \delta$ in $T\left(F_{v}\right)$ put

$$
f_{\varepsilon v}(h)=\Delta_{v}(h) \eta_{v}(\delta) \int_{Z\left(E_{v}\right) G_{\delta}^{o}\left(F_{v}\right) \backslash G\left(E_{v}\right)} \varepsilon_{v}(g) f_{v}\left(g^{-1} \delta \sigma(g)\right) d g .
$$

Here we choose a character $\eta$ of $T(E) \backslash T\left(\mathbf{A}_{E}\right)$ as in Lemma 0.1 , and $\eta_{v}$ is its component at $v$. In particular $f_{\varepsilon v}(h)$ depends only on $h$, and $\eta(\delta)=1$ for all rational $\delta$ in $T(E)$. Put $f_{\varepsilon}(h)=\Pi_{v} f_{\varepsilon v}(h)$ for $h$ in $N T\left(\mathbf{A}_{E}\right)$. Then the $O$-expansion is equal to

$$
\sum_{h \in N T(E) / N Z(E)} f_{\varepsilon}(h) \text {. }
$$

As noted in $\S 0$ we have $N T(E)=N_{L / F_{\varepsilon}} L^{\times}$and $N Z(E)=N_{E / F} E^{\times}$. Moreover, $f_{\varepsilon}(h)=f_{\varepsilon}(\tau h)$ for all $\tau$ in $\operatorname{Gal}\left(F_{\varepsilon} / F\right)$, by definition of $f_{\varepsilon v}$.

14. Identity. Let $V$ be a finite set of places $v$ of $F$. It includes the infinite primes, those which ramify in $F_{\varepsilon} / F$ or $E / F$, where $\eta_{v}$ is ramified, and $\tilde{\Delta}\left(h_{0}\right)$ is not a unit. For $v$ outside $V$ we take $f_{v}$ in the Hecke algebra $\mathbf{H}_{v}$ of $G\left(E_{v}\right)$ with respect to $K_{v}=G\left(\mathcal{O}_{E_{v}}\right)$. Lemma 8.1 implies that $f_{\varepsilon v}=e^{*}\left(f_{v}\right)$ is a spherical function in $\mathbf{H}_{T_{v}}$ on $T\left(F_{v}\right)$. At $v$ in $V$ we take $f_{v}$ which vanishes on the $\delta$ with singular $h=N \delta$. It is clear that $f_{\varepsilon v}$ is then a smooth compactly supported (modulo $N Z\left(E_{v}\right)$ ) function on $N T\left(E_{v}\right)$. It vanishes near the singular set of $T\left(F_{v}\right)$, and transforms under $N Z\left(E_{v}\right)$ by

$$
f_{\varepsilon v}(h N z)=\theta_{v}(z)^{-1} \eta_{v}(z) f_{\varepsilon v}(h)
$$

Each such function on $N T\left(E_{v}\right)$ is so obtained. 
Applying the Poisson summation formula, the $\mathcal{O}$-expansion is equal to $\sum_{\chi} \chi\left(f_{\varepsilon}\right)$, where

$$
\chi\left(f_{\varepsilon}\right)=\prod_{v} \chi_{v}\left(f_{\varepsilon v}\right), \quad \chi_{v}\left(f_{\varepsilon v}\right)=\int_{N Z\left(E_{v}\right) \backslash N T\left(E_{v}\right)} \chi_{v}(h) f_{\varepsilon v}(h) d h .
$$

The sum ranges over all characters $\chi$ of $N T(E) \backslash N T\left(\mathbf{A}_{E}\right)$ which satisfy $\chi(z)=$ $\boldsymbol{\theta}(z) / \eta(z)$ for $z$ in $N Z\left(\mathbf{A}_{E}\right)$. Note that for each $\tau$ in $\operatorname{Gal}\left(F_{\varepsilon} / F\right)$ we have ${ }^{\tau} \chi\left(f_{\varepsilon}\right)=$ $\chi\left(f_{\varepsilon}\right)$, where ${ }^{\tau} \chi(h)=\chi(\tau h)$, since $f_{\varepsilon}(\tau h)=f_{\varepsilon}(h)$. Hence the sum can be put in the form

$$
\sum_{\chi \bmod \operatorname{Gal}\left(F_{\varepsilon} / F\right)} n_{\chi} \chi\left(f_{\varepsilon}\right)
$$

where $n_{\chi}$ denotes the number of $\tau$ in $\operatorname{Gal}\left(F_{\varepsilon} / F\right)$ with ${ }^{\tau} \chi \neq \chi$.

14.1. Proposition. For the above $f$ and $f_{\varepsilon}$ we have

$$
\sum_{\chi} n_{\chi} \chi\left(f_{\varepsilon}\right)=\sum_{M, \tau} c_{M} \operatorname{tr} I(\tau, f \times \sigma \varepsilon) .
$$

The sum on the left ranges over all characters $\chi$ of $N T(E) \backslash N T\left(\mathbf{A}_{E}\right)$ which satisfy $\chi(N z)=\theta(z) / \eta(z)\left(z\right.$ in $\left.Z\left(\mathbf{A}_{E}\right)\right)$, taken modulo $\operatorname{Gal}\left(F_{\varepsilon} / F\right)$. The sum on the right ranges over all divisors $r \geq 1$ of $n$, and all representations $\pi=I_{P}^{G} \tau$ of $G\left(\mathbf{A}_{E}\right)$ with central character $\theta$ induced from a discrete series representation

$$
\tau=\left(\tau^{\prime},{ }^{\sigma} \tau^{\prime} \otimes \varepsilon^{\prime},{ }^{\sigma^{2}} \tau^{\prime} \otimes \varepsilon^{\prime \sigma} \varepsilon^{\prime}, \ldots\right)
$$

of the parabolic subgroup $P\left(\mathbf{A}_{E}\right)$ with Levi factor $M\left(\mathbf{A}_{E}\right)=\operatorname{GL}\left(n^{\prime}, \mathbf{A}_{E}\right)^{r}, n=r n^{\prime}$, with ${ }^{\sigma^{r}} \tau^{\prime}=\tau^{\prime} \otimes \varepsilon^{\prime}{ }^{\sigma} \varepsilon^{\prime} \ldots \sigma^{r-1} \varepsilon^{\prime}$ and $\varepsilon^{\prime}=\varepsilon^{-1}$. The coefficients $c_{M}$ are those of $\S 12$.

15. Refinement. We need a refined form of Proposition 14.1, in which the sums extend over a single element each. For that we prove

15.1. Lemma. Suppose $F$ is local or global, $\varepsilon$ is a character of $C_{E}$ with restriction to $C_{F}$ of order $n$, and $\pi$ is an irreducible admissible or automorphic representation of $\mathrm{GL}(n, E)$ or $\mathrm{GL}\left(n, \mathbf{A}_{E}\right)$ with ${ }^{\sigma} \pi \cong \pi \otimes \varepsilon$. Then there exists a divisor $r$ of $n$, and $a$ (super) cuspidal representation $\tau^{\prime}$ of $\mathrm{GL}\left(n^{\prime}\right)\left(n=r n^{\prime}\right)$ with ${ }^{\sigma^{\prime}} \tau^{\prime} \cong \tau^{\prime} \otimes \varepsilon^{\prime \sigma} \varepsilon^{\prime} \ldots{ }^{\sigma^{r-1}} \varepsilon^{\prime}$ $\left(\varepsilon^{\prime}=\varepsilon^{-1}\right)$, so that $\pi$ is equal to the representation $I_{P}^{G}(\tau)$ induced from the representation

$$
\tau=\left(\tau^{\prime},{ }^{\sigma} \tau^{\prime} \otimes \varepsilon^{\prime},{ }^{\sigma^{2}} \tau^{\prime} \otimes \varepsilon^{\prime \sigma} \varepsilon^{\prime}, \ldots,{ }^{\sigma^{r-1}} \tau^{\prime} \otimes \varepsilon^{\prime \sigma} \varepsilon^{\prime} \ldots{ }^{\sigma^{r-2}} \varepsilon^{\prime}\right)
$$

of the parabolic subgroup $P$ with Levi factor $M=\mathrm{GL}\left(n^{\prime}\right)^{r}$.

Proof. Given $\pi$ there exists a unique (up to association [JS, p. 808]) pair $(P, \tau)$ consisting of a standard (upper triangular) parabolic subgroup $P$ and a cuspidal representation $\tau$ of the Levi subgroup $M$ of $P$, so that $\pi$ is a constituent of the representation $I_{P}^{G}(\tau)$ induced to $G$ from $\tau$ on $P$. The uniqueness is proven in Bernstein-Zelevinski [BZ] if $F$ is local, and in Jacquet-Shalika [JS, Theorem 4.4], for global $F$. Since ${ }^{\sigma} \pi \cong \pi \otimes \varepsilon$ and $\pi$ specifies $I_{P}^{G}(\tau)$, we have $I\left({ }^{\sigma} \tau\right) \cong{ }^{\sigma} I(\tau) \cong I(\tau) \otimes \varepsilon$ $\cong I(\tau \otimes \varepsilon)$, and the argument of $\S 12$ implies that $P$ and $\tau$ have the asserted form. But $I(\tau)$ is irreducible by [BZ], hence $\pi$ is equal to $I(\tau)$, as asserted. 
This lemma implies that all $\tau$ which appear in Proposition 14.1 are not only in the discrete series, but also cuspidal. Since all $\pi=I(\tau)$ of Proposition 14.1 are irreducible, Theorem 4.4 of [JS] implies that for any two such $\pi=I_{P}^{G}(\tau), \pi^{\prime}=I_{P}^{G}\left(\tau^{\prime}\right)$, if $\pi_{v} \cong \pi^{\prime}{ }_{v}$ for almost all $v$, then $\pi \cong \pi^{\prime}$. This fact, its analogue for the $\chi$ 's [K, Proposition 3, p. 238] and the validity of Proposition 14.1 with $f=\otimes f_{v}$ so that $f_{v}$ is an arbitrary spherical function for $v$ outside $V$, implies, via a standard approximation argument [K, p. 242], the following

15.2. Proposition. There exists a one-to-one correspondence from the set of characters $\chi$ of $N T(E) \backslash N T\left(\mathbf{A}_{E}\right)$ with $\chi(N z)=\theta(z) / \eta(z)\left(z\right.$ in $\left.Z\left(\mathbf{A}_{E}\right)\right)$ taken modulo $\operatorname{Gal}\left(F_{\varepsilon} / F\right)$, and automorphic representations $\pi$ of $\operatorname{GL}\left(n, \mathbf{A}_{E}\right)$ with ${ }^{\sigma} \pi \cong \pi \otimes \varepsilon$ (described in Lemma 15.1), and central character $\theta$, so that $\chi\left(f_{\varepsilon}\right)=c \operatorname{tr} \pi(f \times \sigma \varepsilon)$ for all $f, f_{\varepsilon}$ related as in $\$ 14$, where $c$ is a constant independent of $f, f_{\varepsilon}$ (see Proposition 14.1). $F_{\varepsilon}^{\times}$.

Note (as in $\S 0$ ) that the group $N T(E)$ is isomorphic to the subgroup $N_{L / F_{\mathrm{e}}} L^{\times}$of

Notation. We denote the above correspondence by $\chi \leftrightarrow \pi$.

16. We shall now derive a few consequences of Proposition 15.2.

16.1. Corollary. If $\chi$ is a character of $N_{L / F_{\mathrm{e}}} \mathbf{A}_{L}^{\times}$and $\tau^{\prime}$ is a representation of $\mathrm{GL}\left(n^{\prime}, \mathbf{A}_{E}\right)$ with ${ }^{\sigma^{\prime}} \tau^{\prime} \cong \tau^{\prime} \otimes \varepsilon^{\prime \prime}$, where $\sigma^{\prime}=\sigma^{\prime}, \varepsilon^{\prime \prime}=\varepsilon^{\sigma} \varepsilon \cdots \sigma^{\sigma-1} \varepsilon$, so that $\chi$ corresponds to $\tau^{\prime}$, then $\chi$ corresponds to the representation $\pi$ of $\mathrm{GL}\left(n, \mathbf{A}_{E}\right)$ with ${ }^{\sigma} \pi \cong \pi \otimes \varepsilon$, given by

$$
\pi=I_{P}^{G}\left(\tau^{\prime},{ }^{\sigma} \tau^{\prime} \otimes \varepsilon^{\prime},{ }^{2} \tau^{\prime} \otimes \varepsilon^{\prime \sigma} \varepsilon^{\prime}, \ldots,{ }^{\sigma^{\prime-1}} \tau^{\prime} \otimes \varepsilon^{\prime \sigma} \varepsilon^{\prime} \ldots{ }^{\sigma^{\prime-2}} \varepsilon^{\prime}\right),
$$

the representation induced from the parabolic subgroup $P$ with Levi component $M=$ $\mathrm{GL}\left(n^{\prime}\right)$.

Proof. This follows from the validity of the analogous local statement for almost all (unramified) places (see §1), and Proposition 15.2.

16.2. Corollary. Any $\pi$ with $^{\circ} \pi \cong \pi \otimes \varepsilon$ has the form described in Corollary 16.1, locally and globally.

Proof. The global statement follows from Corollary 16.1. To prove the local statement suppose that $\pi=I_{P^{\prime}}^{G}\left(\tau^{\prime \prime},{ }^{\sigma} \tau^{\prime \prime} \otimes \varepsilon^{\prime}, \ldots\right)$, where $\tau^{\prime \prime}$ is a supercuspidal representation of $\mathrm{GL}\left(r, E_{v}\right)$ and $P^{\prime}$ has Levi component $\mathrm{GL}(r)^{s}$, where $r s=n$ and $r$ does not divide $n^{\prime}$. Further ${ }^{\sigma^{\prime \prime}} \tau^{\prime \prime} \cong \tau^{\prime \prime} \otimes \varepsilon^{\prime \prime \prime}$, where $\sigma^{\prime \prime}=\sigma^{s}$ and $\varepsilon^{\prime \prime \prime}=\varepsilon^{\sigma} \varepsilon \ldots \sigma^{s-1} \varepsilon$. To obtain a contradiction, which will prove our assertion, we need to show

16.3. Proposition. If $\pi_{u}$ is a supercuspidal representation of $G\left(E_{u}\right)$ with ${ }^{\sigma} \pi_{u} \cong \pi_{u} \otimes$ $\varepsilon_{u}$, then there exists a cuspidal representation $\pi$ of $G\left(\mathbf{A}_{E}\right)$ with ${ }^{\sigma} \pi \cong \pi \otimes \varepsilon$ and component $\pi_{u}$, where $\varepsilon_{u}$ is the component at $u$ of the character $\varepsilon$ defined in $\$ 10$.

Proof. It suffices to apply the trace formula twisted as in $\$ 10$ with a function $f=\otimes f_{v}$ whose component $f_{u}$ at $u$ is a matrix coefficient of $\pi_{u}$, and its component $f_{w}$ at some $w \neq v$ is supported on the $\sigma$-regular set. For such $f$ the $\chi$-expansion collapses to a sum over cuspidal $\pi$ with ${ }^{\circ} \pi \cong \pi \otimes \varepsilon$ and component $\pi_{u}$ as in the lemma, 
and the $\mathcal{O}$-expansion has the form $\sum_{h} f_{\varepsilon}(h) ; h$ ranges over a discrete subgroup $N Z(E) \backslash N T(E)$ and $f_{\varepsilon}$ is compactly supported. Hence the sum is finite. Reducing the support of $f_{w}$ we may assume that the sum consists of a single summand, and it is nonzero, as required.

16.4. CoROllaRy. For each local $\pi_{u}$ with ${ }^{\sigma} \pi_{u} \cong \pi_{u} \otimes \varepsilon_{u}$ there exists a unique character $\chi_{u}$ of $N_{L_{u} / F_{e u}} L_{u}^{\times}$up to conjugation by $\operatorname{Gal}\left(F_{\varepsilon u} / F_{u}\right)$, so that $\chi_{u}\left(f_{\varepsilon u}\right)=$ $c \operatorname{tr} \pi_{u}\left(f_{u} \times \sigma \varepsilon_{u}\right)$. Here $u$ is a place of $F$ which does not split in $E ; \varepsilon_{u}$ is a character of $E_{u}^{\times}$whose restriction to $F_{u}^{\times}$has order $n$, and it defines the extension $F_{\varepsilon u}$ of $F_{u} . \pi_{u}$ is a representation of $\mathrm{GL}\left(n, E_{u}\right) . c$ is a constant independent of $f_{u}, \pi_{u}$ and $\chi_{u}$.

Proof. Existence follows at once from Lemma 15.1, Proposition 16.3 and Proposition 15.2. Uniqueness follows from the fact that $f_{\varepsilon u}$ is an arbitrary function on the regular set of $N Z\left(E_{u}\right) \backslash N T\left(E_{u}\right)$.

16.5. COROLlaRY. For each $\chi_{u}$ there exists a $\pi_{u}$ as in Corollary 16.4.

PROOF. Extend $\chi_{u}$ to a global character $\chi$, and apply Proposition 15.2.

16.6. Note. It is clear from Corollary 16.1 that the study of the local analogue of the correspondence of Proposition 15.2 can be reduced to the case of $l=1$, namely $F_{\varepsilon} \cap E=F$.

17. It remains to deal with the case where $l=1$, namely $F_{\varepsilon} \cap E=F$.

17.1. PROPOSITION. If $\pi=I(\tau)$, where $\tau=\left(\tau^{\prime},{ }^{\sigma} \tau^{\prime} \otimes \varepsilon^{\prime}, \ldots,{ }^{\sigma^{m-1}} \tau^{\prime} \otimes \varepsilon^{\prime \sigma} \varepsilon^{\prime} \ldots\right.$

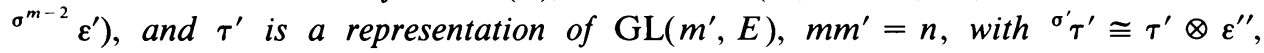
$\sigma^{\prime}=\sigma^{m}, \varepsilon^{\prime \prime}=\varepsilon^{\sigma} \varepsilon \cdots \varepsilon^{m-1}$, then $(m, e)=1$; equivalently $(e, n)$ divides $m^{\prime}$.

Proof. Let $F^{\prime}$ be the fixed field of $\sigma^{\prime}$ in $E$. Its degree over $F$ is the greatest common divisor $(m, e)$ of $m$ and $e$. It is clear that the order of $\varepsilon^{\prime \prime} \mid F^{\prime}$ divides $m^{\prime}$. But $\varepsilon^{\prime \prime} \mid F^{\prime}$ is the $m /(m, e)$ th power of $\varepsilon \circ N_{F^{\prime} / F} \mid F^{\prime}$. As $E \cap F^{\prime}=F$ we deduce that the order of $\varepsilon^{\prime \prime} \mid F^{\prime}$ is $n(m, e) / m=m^{\prime}(m, e)$. This divides $m^{\prime}$ only if $(m, e)=1$.

In this lemma, $\pi$ can be viewed as a local or global representation, and $\varepsilon$ is of course a character of $C_{E}$ whose restriction to $C_{F}$ has order $n$.

In view of Proposition 17.1 we consider only $m^{\prime}$ divisible by $(e, n)$, or $m$ prime to $e$. Let $F_{\varepsilon}^{\prime}$ be an extension of $F$, contained in $F_{\varepsilon}$, of degree $m^{\prime}$. Let $\chi$ be a character of $C_{F_{\varepsilon}}$ which is fixed by $\operatorname{Gal}\left(F_{\varepsilon} / F_{\varepsilon}^{\prime}\right)$. Then there exists a character $\chi^{\prime}$ of $C_{F_{\varepsilon}^{\prime}}$ with $\chi=\chi^{\prime} \circ N_{F_{\varepsilon} / F_{\varepsilon}^{\prime}}$ In fact the restriction of $\chi$ to $N_{L / F_{\varepsilon}} C_{L}$ determines $\chi^{\prime}$ on $N_{L / F_{\varepsilon}} C_{L}$ only. Denote by $\tau^{\prime}$ the representation of $\operatorname{GL}\left(m^{\prime}, E\right)$ with ${ }^{\sigma^{\prime}} \tau^{\prime} \cong \tau^{\prime} \otimes \varepsilon^{\prime \prime}$ corresponding to $\chi^{\prime}$, and put $\pi=I(\tau)$, where $\tau$ is as in Proposition 17.1.

17.2. Proposition. $\chi^{\prime}$ corresponds to $\tau^{\prime}$ if and only if $\chi$ corresponds to $\pi$.

Proof. As this statement is valid in the unramified local case, it follows from Proposition 15.2 that it is true for global representations, and by Proposition 16.3 and Lemma 15.1 it holds locally too. 
Let $F^{\prime}$ be the extension of $F$ of degree $(n, e)$ contained in $F_{\varepsilon}$. It follows that

17.3. COROLLARY. $\chi$ corresponds to a (super) cuspidal $\pi$ if and only if $\chi$ is not fixed by any element of $\operatorname{Gal}\left(F_{\varepsilon} / F^{\prime}\right)$.

Note that our local correspondence $\chi \leftrightarrow \pi$ can be expressed also by means of character relations, thus determining germs of certain linear combinations of characters, as in [K, Theorem A(a), p. 212]. However, the factor $\Delta\left(l^{\sigma}\right)$ there should be $\Delta(l)$ (independent of $\sigma$ ). This is useful for the study of the metaplectic correspondence.

There remains to show that locally the correspondence $\chi \leftrightarrow \pi$ is one-to-one. By Corollary 17.3 it suffices to show this for supercuspidal $\pi$. Namely we have to show

17.4. Proposition. If $\pi$ and $\pi^{\prime}$ are supercuspidal and

$$
\operatorname{tr} \pi(f \times \sigma \varepsilon)=c \operatorname{tr} \pi^{\prime}(f \times \sigma \varepsilon),
$$

then $\pi$ and $\pi^{\prime}$ are equivalent.

Proof. It suffices to let $f$ be a matrix coefficient $f_{\pi}$ of $\pi$, for then the left side is 1 , and the right side is 0 unless $\pi \cong \pi^{\prime}$. However, the identity (*) is available for functions $f$ supported on the $\sigma$-regular set only, by our assumptions in $\S 10$. So it remains to show that (*) holds also with $f=f_{\pi}$. But the characters $\chi_{\pi}$ and $\chi_{\pi^{\prime}}$ can be shown by methods of Harish-Chandra $[\mathbf{H}]$ to be locally integrable on $G(E)$. Hence the functional $f \mapsto \operatorname{tr} \pi(f \times \sigma \varepsilon)$ is continuous. Since $f_{\pi}$ is a limit of functions $f$ supported only on the $\sigma$-regular set, the lemma follows.

17.5. We wish to present an alternative proof for the fact, used in Proposition 17.4 , that there exists a locally integrable function $\chi_{\pi}(g)$ on $G(E)$, smooth on the set of $g$ with regular $\mathrm{Ng}$, so that

$$
\operatorname{tr} \pi(f \times \sigma \varepsilon)=\int_{Z(E) \backslash G(E)} \chi_{\pi}(g) f(g) d g .
$$

(Our equalities below are valid only up to a constant multiple.) Indeed,

$$
\begin{aligned}
\operatorname{tr} \pi( & f \sigma \varepsilon)=\chi\left(f_{\varepsilon}\right) \\
= & \int_{Z(E) T(E)^{1-\sigma} \backslash T(E)} \chi(N \delta) \Delta(N \delta) \eta(\delta) \int_{G_{\delta}^{g}(E) \backslash G(E)} \varepsilon(g) f\left(g^{-1} \delta \sigma(g)\right) \\
= & \int_{\left[Z(E) T(E)^{1-\sigma} \backslash T(E)\right] \times\left[G_{\delta}^{g}(E) \backslash G(E)\right]} \Delta(N \delta)^{2} \\
& \times\left[\sum_{G a l\left(F_{\varepsilon} / F\right)} \Delta\left(N \delta^{w}\right)^{-1} \chi\left(N \delta^{w}\right) \eta\left(\delta^{w}\right) \varepsilon(g)\right] f\left(g^{-1} \delta \sigma(g)\right) .
\end{aligned}
$$

Hence it follows from the Weyl integration formula that

$$
\chi_{\pi}\left(g^{-1} \delta \sigma(g)\right)=\varepsilon(g) \sum \chi\left(N \delta^{w}\right) \eta\left(\delta^{w}\right) / \Delta\left(N \delta^{w}\right)
$$


if $\delta$ lies in $T(E)$, and $\chi_{\pi}(g)=0$ if $N g$ is regular but not conjugate to an element of $T(F)$, as claimed.

17.6. From 17.5 it follows that

$$
\chi_{\pi}\left(g^{-1} \delta \sigma(g)\right)=\varepsilon(g) \chi_{\pi}(\delta) \quad(\delta \text { in } T(E)) .
$$

We can arrive at this formula without the explicit computation of $\chi_{\pi}$. Recall that ${ }^{\circ} \pi \cong \pi \otimes \varepsilon$, hence $A=\pi(\sigma \varepsilon)$ is an intertwining operator with $A \pi(g)=$ $\pi(\sigma g) \varepsilon^{-1}(g) A$. Also $\pi(f)=\int f(x) \pi(x) d x$, hence

$$
\begin{aligned}
\pi\left(g^{-1}\right) \pi(f) \pi(\sigma g) & =\int f(x) \pi\left(g^{-1} x \sigma g\right)=\int f\left(g x \sigma g^{-1}\right) \pi(x) \\
& =\int f^{g}(x) \pi(x)=\pi\left(f^{g}\right),
\end{aligned}
$$

where $f^{g}(x)=f\left(g x \sigma g^{-1}\right)$. Hence

$$
\begin{aligned}
\int \chi_{\pi}(x) f(x) & =\operatorname{tr} \pi(f) A=\operatorname{tr} \pi(g)^{-1} \pi(f) A \pi(g) \\
& =\operatorname{tr} \pi\left(g^{-1}\right) \pi(f) \pi(\sigma g) \varepsilon(g)^{-1} A \\
& =\operatorname{tr} \varepsilon(g)^{-1} \pi\left(f^{g}\right) A=\varepsilon(g)^{-1} \int f\left(g x \sigma g^{-1}\right) \chi_{\pi}(x) \\
& =\varepsilon(g)^{-1} \int \chi_{\pi}\left(g^{-1} x \sigma g\right) f(x)
\end{aligned}
$$

as asserted.

17.7. Finally we give an alternative proof for the validity of (*) with $f=f_{\pi}$. Theorem 9 of $[\mathbf{H}$, p. 43$]$ asserts that

$$
\begin{aligned}
\operatorname{tr} \pi(f) A & =\int_{Z(E) \backslash G(E)} d g \int_{G(E)} f(x)\left(v, \pi\left(g^{-1}\right) \pi(x) A \pi(g) v\right) d x \\
& =\int d g \int d x \varepsilon^{-1}(g) f(x)\left(v, \pi\left(g^{-1} x \sigma g\right) A v\right),
\end{aligned}
$$

where $(\cdot, \cdot)$ is an inner product in the space of $\pi$ and $v$ is a vector of length 1 . We put $\tilde{f}_{\pi}(g)=(v, \pi(g) A v)$. Part $\mathrm{V}$ of $[\mathbf{H}]$ (suitably modified to our twisted case), implies that since $\pi$ is supercuspidal, we have

$$
\chi_{\pi}(\delta)=\int_{G_{\delta}^{\sigma}(E) \backslash G(E)} \varepsilon(x)^{-1} \tilde{f}_{\pi}\left(x^{-1} \delta \sigma x\right) .
$$

Hence if we put $f_{\pi}(g)=(\pi(g) A v, v)$, it follows from the explicit determination of $\chi_{\pi}$ above that $\Delta(N \delta) \int \varepsilon(x) f_{\pi}\left(x^{-1} \delta \sigma x\right)$ is a smooth function of $\delta$. Hence

$$
\operatorname{tr} \pi^{\prime}\left(f_{\pi} \times \sigma \varepsilon\right)=\int \chi_{\pi^{\prime}}(g) f_{\pi}(g)=\int \chi(N \delta) \Delta(N \delta) \eta(\delta) \int \varepsilon(g) f_{\pi}\left(g^{-1} \delta \sigma g\right)
$$


( $\delta$ in $Z(E) T(E)^{1-\sigma} \backslash T(E), g$ in $G_{\delta}^{\sigma}(E) \backslash G(E)$ ), is a well-defined convergent integral, which is equal to $\operatorname{tr} \pi\left(f_{\pi} \times \sigma \varepsilon\right)(=1)$, and $(*)$ holds with $f=f_{\pi}$, as was to be shown.

Proposition 17.4 has the following generalization.

18. Proposition. The characters $f \rightarrow \operatorname{tr} \pi(f \times \sigma \varepsilon)$ of a finite set of inequivalent representations $\pi$ of $G^{\prime}=\mathrm{GL}(n, E)$ are linearly independent.

Proof. Since ${ }^{\sigma} \pi \simeq \pi \otimes \varepsilon$, there eixsts an operator $A$ on the space of $\pi$ with $A \pi(\sigma x)=\varepsilon(x) \pi(x) A$. Hence $A^{e} \pi(x)=\varepsilon(N x) \pi(x) A^{e}$, and the order $n^{\prime}$ of $\varepsilon(N x)$ divides $n$. Thus $A^{e n^{\prime}} \pi(x)=\pi(x) A^{e n^{\prime}}$ for all $x$ in $G^{\prime}$, and $A^{e n^{\prime}}$ is a scalar which we can choose to be 1 . Let $G^{\prime \prime}$ be the direct product of $\mu_{n}$ with the semidirect product $G^{\prime} \times\langle\sigma \varepsilon\rangle$. Here $\langle\sigma \varepsilon\rangle$ is the cyclic group of order $e n^{\prime}$ generated by the symbol $\sigma \varepsilon$, and $(\sigma \varepsilon)^{-1} g \sigma \varepsilon=\left(\sigma(g), \varepsilon(g)^{-1}\right)$. Then $\pi$ extends to a representation $\pi^{\prime}$ of $G^{\prime \prime}$ which is faithful on the $\mu_{n}$ factor, and $\pi^{\prime}(\sigma \varepsilon)=A$. Let $\tau$ be a character of $\langle\sigma \varepsilon\rangle$ so that $\zeta=\tau(\sigma \varepsilon)$ is a primitive $e n^{\prime}$ th root of unity. Put $\pi_{i}^{\prime}=\pi^{\prime} \otimes \tau^{i}$. Then

$$
\left(e n^{\prime}\right)^{-1} \sum_{i} \tau^{-i}(\sigma \varepsilon) \chi_{i}^{\prime}\left(x \times(\sigma \varepsilon)^{j}\right)=\delta_{j 1} \chi(x \times \sigma \varepsilon)
$$

where

$$
\operatorname{tr} \pi_{i}^{\prime}\left(f \times(\sigma \varepsilon)^{j}\right)=\int_{G^{\prime}} \chi_{i}^{\prime}\left(x \times(\sigma \varepsilon)^{j}\right) f(x) d x
$$

Now a linear relation of the $\chi(x \times \sigma \varepsilon)$ implies a linear relation of the $\chi_{i}^{\prime}$. But if $\pi$ and $\tilde{\pi}$ are inequivalent then $\pi_{i}^{\prime}$ and $\tilde{\pi}_{j}^{\prime}$ are inequivalent for all $i$ and $j$, and we obtain a contradiction by linear independence of characters on the group $G^{\prime \prime}$.

Let $\psi=\psi^{E / F}$ be a character of $F^{\times}$whose kernel is $N_{E / F} E^{\times}$, and $\theta$ the characteristic function of $x$ in $G=\mathrm{GL}(n, F)$ with $\psi(\operatorname{det} x)=1$.

COROLlaRY. Let $\pi_{i}$ be finitely many irreducible representations of $G$ with $\pi_{i} \otimes \psi^{j}$ inequivalent to $\pi_{k}$ for all integers $i, j, k$. Then the characters $f \rightarrow \operatorname{tr} \pi_{i}(\theta f \times \varepsilon)$ are linearly independent.

ProOF. $e \operatorname{tr} \pi(\theta f \times \varepsilon)=\int \chi_{\pi}(x)\left[\sum_{i} \psi^{i}(x)\right] f(x) d x=\sum_{i} \operatorname{tr} \pi \otimes \psi^{i}(f \times \varepsilon)$.

Note that $\operatorname{tr} \pi \otimes \psi(\theta f \times \varepsilon)=\operatorname{tr} \pi(\theta f \times \varepsilon)$.

\section{REFERENCES}

[A] J. Arthur, A trace formula for reductive groups. I, Duke Math. J. 45 (1978), 911-952.

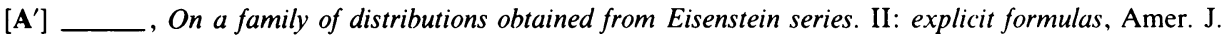
Math. 104 (1982), 1289-1336.

[BZ] J. Bernstein and A. Zelevinsky, Induced representations of reductive p-adic groups, Ann. Sci. Ėcole Norm. Sup. (4) 10 (1977), 441-472.

[B] A. Borel, Automorphic L-functions, Proc. Sympos. Pure Math., vol. 33, II, Amer. Math. Soc., Providence, R. I., 1979, pp. 27-63.

[C] P. Cartier, Representations of p-adic groups, Proc. Sympos. Pure Math., vol. 33, I, Amer. Math. Soc., Providence, R. I. 1979, pp. 111-157.

[F] Y. Flicker, Stable and labile base change for U(2), Duke Math. J. 49 (1982), 691-729. 
[H] Harish-Chandra, Harmonic analysis on reductive p-adic groups, (notes by G. van Dijk), Lecture Notes in Math., vol. 162, Springer-Verlag, Berlin and New York, 1970. See also: Admissible invariant distributions on reductive p-adic groups, Queen's Papers in Pure and Applied Math. 48 (1978), 281-346.

[JS] H. Jacquet and Shalika, On Euler products and the classification of automorphic forms. II, Amer. J. Math. 103 (1981), 777-815.

[K] D. Kazhdan, On lifting, Lie Group Representations. II, Lecture Notes in Math., vol. 1041, Springer-Verlag, Berlin and New York, 1984, pp. 209-249.

[Ko] R. Kottwitz, Base change transfer of unit element in Hecke algebra, Lecture at IAS, 1984.

[T] J. Tate, Number theoretic background, Proc. Sympos. Pure Math., vol. 33, II, Amer. Math Soc., Providence, R. I., 1979, p. 3-27.

[Ti] J. Tits, Reductive groups over local fields, Proc. Sympos. Pure Math., vol. 33, I, Amer. Math. Soc., Providence, R.I., 1979, pp. 29-69.

Department of Mathematics, Princeton University, Princeton, New Jersey 08544

Current address: Department of Mathematics, Harvard University, Science Center, 1 Oxford Street, Cambridge, Massachusetts 02138 